\title{
PARANEOPLASTIC SYNDROME IN LUNG CANCER
}

\author{
Yu.V. Dumansky', O.V. Syniachenko ${ }^{1}$, P.A. Stepko ${ }^{1}$, Ye.D. Yehudina ${ }^{2}$, O.Yu. Stoliarova ${ }^{3}$ \\ ${ }^{1}$ Donetsk National Medical University, Lyman 8440, Ukraine \\ ${ }^{2}$ State Institution «Dnipropetrovsk State Medical Academy of the Ministry of Health of Ukraine», \\ Dnipro 49044, Ukraine \\ ${ }^{3}$ National Cancer Institute, Kyiv 03022, Ukraine
}

\begin{abstract}
Aim: To study the nature of different variants of paraneoplastic syndrome (PNPS) in lung cancer, taking into account the features of the tumorous process and the complications of radiochemotherapy. Patients and Methods: We performed an analysis of the data of 1,669 patients with lung cancer aged between 24 and 87 years, among whom there were $89 \%$ of men and $11 \%$ of women. The ratio of small cell and non-small-cell histological variants of the lung cancer was 1:4, IB, IIA, IIB, IIIA, IIIB and IV stages of cancer 1:2:6:58:43:57. Results: PNPS developed in 16\% of the lung cancer patients, in these patients we have detected a marked increase in the disease incidence in women, the peripheral form of the tumor, the apical variant of Pancoast - Tobias and adenocarcinoma, but no cases of the median lower localization of the tumor. The number of the upper lobar pathology was decreased, while the severity of the cancer was significantly greater, which more often occurred with exudative pleurisy, germination of the tumor into the chest wall and compression of the upper vena cava. The 21 components of PNPS pathology were established. We distributed them conditionally into the musculoskeletal system lesions, variants of skin vasculitis and autoimmune processes, the nature of which depended on the localization and course of the tumorous process, its histological variation and severity of the course. Moreover, PNPS negatively affected the development of radiochemotherapy complications and worsened survival rate. Conclusions: The course of PNPS in lung cancer is highly diverse, being a risk factor for a severe tumorous process that worsens the survival of patients. Key Words: lung cancer, paraneoplastic syndrome.
\end{abstract}

Lung cancer (LC) has the leading position in the structure of oncological morbidity, resulting in annual mortality increase in an average of $10 \%$ in some regions [1-3]. LC (especially its small cell histological variant and adenocarcinoma) is the most common cause of the so-called paraneoplastic syndrome (PNPS) development [4], which is not directly related to the primary neoplasm and its metastases, but it is due to the complex immune-inflammatory, degenerative and vascular distant changes $[5,6]$. The study of the PNPS course peculiarities in LC has become one of the most urgent modern oncology problems [7].

PNPS, as a rule, proceeds with rheumatological, dermatological, endocrinological, neurological and nephrological manifestations [8-11]. Miret et al. [12] found that autoimmune paraneoplastic disorders occurred in patients with non-small cell and small cell LC in a ratio of 1:9. Osteoarthropathy, arthritis, myositis and other autoimmune syndromes occur in $15 \%$ of the LC patients [13], more often in patients with bone metastases [14]. It should be noted that the successful treatment of $L C$ (surgical, radiochemotherapy) may be accompanied by the disappearance of PNPS signs [15]. The prognostic value of the latter in patients with LC remains inadequate studied [16, 17].

The aim of this study was to evaluate the nature of different PNPS variants in LC, taking into account

Submitted: May 16, 2018.

*Correspondence: E-mail: oncologdopc@gmail.com Abbreviations used: IRCT - intensity of radio- and chemotherapy; ITDG - index of tumor differentiation; LC - lung cancer; PNPS paraneoplastic syndrome; $\mathrm{SI}$ - severity index of the tumorous process; StT - stage of the tumor. the peculiarities of the tumor process course and the radiochemotherapy complications.

\section{PATIENTS AND METHODS}

The work was performed in accordance with the ethical standards set forth in the Declaration of the Helsinki Medical Assembly, and the patients gave their informed consent to a study approved by the Donetsk National Medical University Bioethics Commission.

The material of 4 oncological Ukrainian centers was retrospectively analyzed. There were 1669 patients with LC aged from 24 to 87 ( $59.4 \pm 0.24$ years on average), among whom $88.8 \%$ were men and $11.2 \%$ were women. Right-sided localization of the cancer process occurred in $56.9 \%$ of the patients, left-sided $-41.8 \%$, bilateral $-1.3 \%$. The upper lobe LC was diagnosed in $28.6 \%$ of the cases, the middle-upper lobe $21.3 \%$, the left upper lower lobe $-20.2 \%$, the lower lobe $-13.1 \%$, the mediastinal $-11.0 \%$, the median lobe $-2.9 \%$, the median lower lobe $-1.7 \%$. The small cell histological variant of the disease was diagnosed in $18.5 \%$ of the observations, and non-small-cell in $81.6 \%$, among which adenocarcinoma was detected in $37.2 \%$ of the total number of the examined patients and in $45.6 \%$ of the non-small cell form, squamous cell carcinoma - in $35.2 \%$ and $43.2 \%$, respectively, large-cell carcinoma - in 9.2 and $11.2 \%$.

IB stage of the disease was detected in $0.6 \%$ of LC cases, IIA $-1.3 \%$, IIB $-3.6 \%$, IIIA $-34.8 \%$, IIIB $-25.9 \%$, IV $-33.9 \%$. The average index of LC differentiation (GDT) was $1.160 \pm 0.030$ points, the integral stage index (StT) was $5.850 \pm 0.025$ r.u., the tumorous process severity index ( $\mathrm{SI}$ ) was $2.850 \pm 0.019$ a.u., which was estimated by the formula:

$$
S I=\ln \left[T+N^{2}+(\Sigma M)^{2}\right]
$$


where $I n$ is a natural logarithm; $T$ is an international indicator of the primary tumor extent; $N$ is the international index of regional lymph nodes metastatic lesion; $\Sigma M$ is the sum of metastases in distant organs.

Among the manifestations of LC, plevral exudates were observed in $9.7 \%$ of patients, compression syndrome - in $7.5 \%$, the germination of the tumor into the trachea - in $6.8 \%$, into the ribs - in $2.8 \%$, into the chest - in $2.5 \%$, into the esophagus - in $2.3 \%$ and into the pericardium - in $0.8 \% ; 4.0 \%$ of the patients had obstructive atelectasis; $3.1 \%$ had the compression of the recurrent nerve; and $1.6 \%$ - the upper vena cava compression. Metastases to the lymph nodes were found in $81.1 \%$ of LC cases, metastases in distant organs - in $30.7 \%$, in the skeleton - in $20.0 \%$. For the diagnosing of the disease we used such apparatus as "Multix-Compact-Sièmens" (Germany), "SomazomEmotion-6-Siemens" (Germany), "Gygoscan-InteraPhilips" (Netherlands), "Siemens-RDS-Eclipse-RD" cyclotron (Germany), "Olympus-GIF-Q20" (Japan) fiberscope and "Envisor-Philips" (Netherlands) sonograph.

Statistical processing of the obtained results was performed using computer variance, nonparametric, correlation, regression, single (ANOVA) and multivariate (ANOVA/MANOVA) dispersion analysis (Microsoft Excel and Statistica-Stat-Soft, USA programs). We estimated the average values (M), their standard errors and deviations (SD), the parametric correlation coefficients of Pearson and the nonparametric of Kendall, the criteria of Brown - Forsythe and Wilcoxon - Rao dispersion, Student and McNemar - Fisher's test $\left(X^{2}\right)$, and the reliability of the statistical indices $(p)$. The critical significance level for checking the statistical hypotheses was considered to be 0.05 .

In assessing the results of radiochemotherapy, the treatment intensity (IRCT) was studied. Thus, the average dosages of drugs and radiation $\{<M+S D\}$ were defined as minimum in 1 point, the dosages $\{M+S D \div$ $M+2 S D\}-$ as moderate in 2 points, $\{M+2 S D \div$ $M+3 S D\}-$ as high in 3 points and $\{>M+3 S D\}-$ as very high in 4 points. The treatment intensity $W$ was calculated by the formula:

$$
W=(a+b+c+d) / n,
$$

where $a, b, c, d-$ the number of radiation zones or drugs in 1, 2, 3 and 4 points, respectively; $n$ is the total number of radiation zones or the total number of used drugs.

\section{RESULTS AND DISCUSSION}

Among 1,669 examined patients with LC, the main $\left(1^{\text {st }}\right)$ group consisted of $258(15.5 \%)$ patients aged from 24 to 79 years ( $58.6 \pm 0.65$ years old on average), in which certain signs of PNPS were revealed. The comparison group $\left(2^{\text {nd }}\right)$ comprised from the remaining 1411 patients. In addition, the patients of the main group were divided into 3 subgroups $-10.4 \%$ of the total number and $67.1 \%$ of the number with PNPS associated with bone-articular system and periarticular tissue lesions (subgroup A); $4.7 \%$ and $30.6 \%$ patients, respectively - with different variants of skin vasculitis (subgroup B); and $4.7 \%$ and $30.2 \%$ - with systemic autoimmune processes (subgroup $\mathrm{C}$ ).

The first group consisted of $79.9 \%$ men and $20.2 \%$ women, the $2^{\text {nd }}-90.4 \%$ men and $9.6 \%$ women. Thus, the ratio of men and women in the $1^{\text {st }}$ group was 4:1, and in the $2^{\text {nd }}-9: 1$ (the differences were significant). The patients' average age in these groups was slightly different. The $1^{\text {st }}$ and $2^{\text {nd }}$ groups were approximately the same on the frequency of the side lesions by the tumor process, while the ratio of the central and peripheral forms was 3:1 in the main group and 5:1 in the control group. In the group of the PNPS patients, there were no patients with a tumor process localizing in the right median lower lobe, but they were diagnosed apical Pancoast - Tobias LC 4.4 times as often. In this regard, the tumor of Pancoast - Tobias can be assumed to be a risk factor for the development of PNPS, and the prognosisfavorable factor in this respect is tumor localization in the right median lower lobe. Patients of the main group have adenocarcinoma more often (8\%). Patients of the $1^{\text {st }}$ and $2^{\text {nd }}$ groups did not differ by such histological forms of non-small cell $L C$ as squamous and large-squamous.

Subgroup A consisted of $24.0 \%$ of the PNPS patients with Marie - Bamberger osteoarthropathy, $22.9 \%$ - with gouty arthritis, $15.9 \%$ - with paraneoplastic rheumatoid-like reactive arthritis, $14.3 \%$ - with peripheral neuropathy, $5.8 \%$ - with palmar-plantar fibrositis and tendovaginitis respectively, 3,5\%with fasciitis and enthesopathy, $3.1 \%$ - with algodystrophy. Subgroup B included $10.5 \%$ of patients with leukocytoclastic vasculitis, $10.1 \%$ - with panniculitis and nodular erythema, $8.5 \%$ - with digital arteritis, $1.9 \%$ - with cryoglobulinemic vasculitis, and subgroup $\mathrm{C}-12.4 \%$ with dermatopolymyositis, $6 . \%$ with scleroderma-like syndrome, $4.7 \%$ - with lupus-like syndrome, $4.3 \%$ - with limbic encephalitis, $3.9 \%$ - with glomerulonephritis (membranous, mesangiocapillary), 3.5\% - with myasthenic Lambert Eaton syndrome, $2.3 \%$ - with Kikuchi - Fujimoto necrotizing lymphadenitis syndrome, $1.9 \%$ - with Sjogren's syndrome). For one patient with PNPS there were from 1 to 9 symptoms (on average $1.77 \pm 0.090$ ).

Patients in the main group had the significantly increased StT (9\%) and SI (1/4) indices. At the same time (Table 1 ), the $1^{\text {st }}$ group significantly differed from the $2^{\text {nd }}$ group by the higher frequency of exudative pleurisy (2.1 times), tumor growth into the thorax (3.2 times), and the compression of the upper vena cava (4.7 times).

According to Wilcoxon - Rao multivariate dispersion analysis, clinical signs of LC course influenced on the nature of PNPS. We selected those signs of LC clinical course that simultaneously had Brown Forsyth and correlation Kendall dispersion relations with PNPS. It turned out that the number of constituent manifestations of PNPS rises with the age of LC patients, with an increase in the degree and severity of the disease course. All subgroups of patients with PNPS $(A, B, C)$ are affected by the localization of the tumor process, the form of LC, the squamous histological variant, the parameters of StT and SI. 
Table 1. Frequency of $L C$ separate manifestations in patients of the $1^{\text {st }}$ and $2^{\text {nd }}$ groups

\begin{tabular}{ccccccc}
\hline \multirow{3}{*}{ LC signs } & \multicolumn{3}{c}{ Patients groups } & \multicolumn{3}{c}{$\begin{array}{c}\text { Differences between } \\
\text { the groups }\end{array}$} \\
\cline { 2 - 5 } & $1^{\text {st }}(\mathrm{n}=258)$ & \multicolumn{2}{c}{$2^{\text {nd }}(\mathrm{n}=1411)$} & \multicolumn{2}{c}{$\chi^{2}$} & $p$ \\
\cline { 2 - 5 } & $\mathrm{n}$ & $\%$ & $\mathrm{n}$ & $\%$ & 19.21 & $<0.001$ \\
2 & 44 & 17.1 & 117 & 8.3 & 0.12 & 0.734 \\
3 & 18 & 7.0 & 107 & 7.6 & 2.13 & 0.144 \\
4 & 6 & 2.3 & 60 & 4.3 & 0.91 & 0.341 \\
5 & 21 & 8.1 & 92 & 6.5 & 0.72 & 0.395 \\
6 & 4 & 1.6 & 34 & 2.4 & 14.36 & $<0.001$ \\
7 & 15 & 5.8 & 26 & 1.8 & 0.01 & 0.903 \\
8 & 2 & 0.8 & 12 & 0.9 & 0.14 & 0.713 \\
9 & 8 & 3.1 & 38 & 2.7 & 0.001 \\
10 & 12 & 4.7 & 14 & 1.0 & 19.04 & $<0.001$ \\
\hline
\end{tabular}

Note: 1 - exudative pleurisy, 2 - compression syndrome, 3 - obturation atelectasis, 4 - tumor germination into the trachea, 5 - tumor germination into the esophagus, 6 - tumor germination into the thoracic wall, $7-$ tumor germination into the pericardium, 8 - tumor germination into the rib, 9 - tumor compression of the superior vena cava, 10 - tumor compression of the recurrent nerve.

According to Brown - Forsythe dispersion analysis, the number of PNPS manifestations in one patient with LC is affected by the germination of the tumor into the esophagus, the chest wall, and the upper vena cava. In subgroup A, such an effect concerns the LC germination into the rib, in subgroup $B$ - germination into the trachea, esophagus, thoracic wall and the upper vena cava; in subgroup C, PNPS is associated with the presence of exudative pleurisy and tumor germination into the pericardium.

PNPS in the form of the musculoskeletal system lesions has a dispersion and direct correlation with metastases into the skeleton, PNPS caused by autoimmune pathology - with metastases to distant organs. Among the concomitant diseases, the development of PNPS is closely related to the presence of chronic bronchitis, chronic obstructive pulmonary disease, diabetes mellitus, chronic gastroduodenitis and ulcers of the stomach and duodenum in patients with LC.

It should be noted that PNPS in LC patients may result from the chemotherapy complications [18], which primarily concerns platinum-based and taxane agents [19]. As shown by Brown - Forsythe dispersion analysis and Kendall nonparametric correlation, the power of the performed radiation therapy, the usage of 8 groups of drugs included in chemotherapy such as anti-metabolites (gemcitabine, methotrexate, pemetrexed, fluorouracil), vinca alkaloid (vinblastine, vincristine), anthracyclines (bleomycin, doxorubicin, idarubicin, mitomycin, epirubicin) have a direct negative effect on the formation of PNPS in LC.

It should benoted that in the main group of $L C$ patients, the IRCT was $1.470 \pm 0.054$ and $0.400 \pm 0.026$ a.u., whereas in the PNPS absence, they were significantly less by $30 \%$ and $25 \%$ respectively. In $1^{\text {st }}$ group, the number of PNPS signs was directly related to both the power of radiation and chemotherapy, as demonstrated by the dispersion and correlation analysis.

In the process of radiochemotherapy, myelodepression developed in $7.0 \%$ of cases, acute radiation pneumonitis $-3.0 \%$, pulmonary hypertension $2.9 \%$, radiation osteophagitis $-2.2 \%$, acute coronary syndrome $-2.0 \%$, radiation lung fibrosis and indomi- table vomiting $-1.6 \%$, acute tubulointerstitial nephritis $-1.4 \%$, stroke $-1.3 \%$, acute thrombophlebitis $1.2 \%$, persistent diarrhea $-0.8 \%$, jaundice $-0,7 \%$, pulmonary artery thromboembolism $-0.6 \%$ (Table 2 ).

The multivariate analysis of Wilcoxon - Rao demonstrated the effect of the PNPS nature on the complications of radiochemotherapy. According to McNemar Fisher non-parametric comparative analysis, in the presence of PNPS, pulmonary hypertension developed significantly 2.3 times, cerebral circulation lesion -3.4 times, radiation pneumophilia -3.6 times, jaundice -4.8 times, thrombophlebitis -7.8 times as often.

Table 2. The frequency of separate complications caused by therapeutic interventions (radiochemotherapy) in LC patients of the $1^{\text {st }}$ and $2^{\text {nd }}$ groups

\begin{tabular}{ccccccc}
\hline \multirow{2}{*}{ LC signs } & \multicolumn{3}{c}{ Patients groups } & \multicolumn{2}{c}{ Differences } \\
\cline { 2 - 5 } & \multicolumn{2}{c}{$1^{\text {st }}(\mathrm{n}=258)$} & \multicolumn{2}{c}{$2^{\text {nd }}(\mathrm{n}=1411)$} & \multicolumn{2}{c}{ between the groups } \\
\cline { 2 - 5 } & $\mathrm{n}$ & $\%$ & $\mathrm{n}$ & $\%$ & $\mathrm{X}^{2}$ & $\mathrm{p}$ \\
\hline 1 & 21 & 8.1 & 96 & 6.8 & 0.37 & 0.543 \\
2 & 11 & 4.3 & 39 & 2.8 & 1.69 & 0.194 \\
3 & 14 & 5.4 & 34 & 2.4 & 7.11 & 0.008 \\
4 & 8 & 3.1 & 29 & 2.1 & 1.10 & 0.294 \\
5 & 6 & 2.3 & 28 & 2.0 & 0.13 & 0.721 \\
6 & 10 & 3.9 & 16 & 1.1 & 10.69 & 0.001 \\
7 & 3 & 1.2 & 23 & 1.6 & 0.31 & 0.577 \\
8 & 7 & 2.7 & 17 & 1.2 & 3.50 & 0.061 \\
9 & 8 & 3.1 & 13 & 0.9 & 8.34 & 0.004 \\
10 & 12 & 4.7 & 8 & 0.6 & 30.73 & $<0.001$ \\
11 & 4 & 1.6 & 9 & 0.6 & 2.35 & 0.125 \\
12 & 5 & 1.9 & 6 & 0.4 & 7.62 & 0.006 \\
13 & 4 & 1.6 & 6 & 0.4 & 2.43 & 0.119 \\
\hline
\end{tabular}

Note: 1 - myelodepression, 2 - acute radiation pneumonitis, 3 - pulmonary hypertension, 4 - radiation esophagitis, 5 - acute coronary syndrome, 6 - radiation pulmonary fibrosis, 7 - indomitable vomiting, 8 - acute tubulointerstitial nephritis, 9 - stroke, 10 - acute thrombophlebitis, 11 - persistent diarrhea, 12 - jaundice, 13 - pulmonary artery branches embolism.

The three-year survival indicators of the $1^{\text {st }}$ group patients turned out to be worse (Figure) and all patients with PNPS died in 30 months. In deceased patients, life expectancy after diagnosis of $\mathrm{LC}$ was on average by 4 months less, being $10.8 \pm 0.31$ months. As shown by the dispersion analysis, the life span was influenced by the number of PNPS components in one such patient.

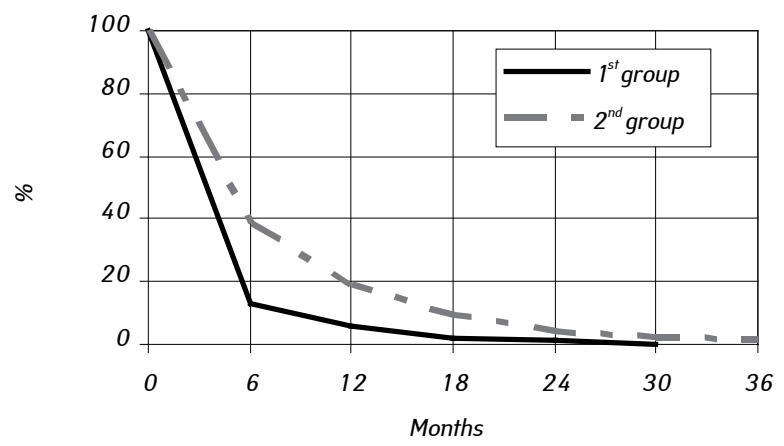

Figure. Three-year survival rates of the patients from the $1^{\text {st }}$ and $2^{\text {nd }}$ groups

\section{CONCLUSION}

PNPS developed in $16 \%$ of the LC patients, in these patients we have detected a marked increase in the incidence of the disease in women, the peripheral form of the tumor, the Pancoast - Tobias apical variant and adenocarcinoma, but no cases of the median lower 
localization. The number of the upper lobar pathology was decreased, while the severity of the cancer was significantly greater, which more often occurred along with exudative pleurisy, germination of the tumor into the chest wall and compression of the upper vena cava. The 21 components of PNPS pathology were established, we distributed them conditionally into the musculoskeletal system lesions, variants of skin vasculitis and autoimmune processes, the nature of which depends on the localization and course of the tumorous process, its histological variation and course severity. Moreover, PNPS negatively affected the development of radiochemotherapy complications and worsened survival rate.

\section{REFERENCES}

1. Gradalska-Lampart M, Karczmarek-Borowska B, Radziszewska AU. Lung cancer in Podkarpackie region in the years 2002-2011. Pneumonol Alergol Pol 2015; 83: $109-19$.

2. Latimer KM. Lung cancer: clinical presentation and diagnosis. FP Essent 2018; 464: 23-6.

3. Qu HM, Bai YN, Cheng Y, et al. Trend analysis of cancer mortality in the jinchang cohort, China, 2001-2010. Biomed Environ Sci 2015; 28: 364-9.

4. Wilkins CM, Johnson VL, Fargason RE, Birur B. Psychosis as a sequelae of paraneoplastic syndrome in small-cell lung carcinoma: A psycho-neuroendocrine interface. Clin Schizophr Relat Psychoses 2017; 22: 153-8.

5. Hébant B, Miret N, Berthelot L, et al. Generalized pruritus preceding paraneoplastic neuropathy. J Clin Neurosci 2016; 26: 156-7.

6. Ramírez-Bellver JL, Macías E, Bernárdez C, et al. Anti-NXP2-positive paraneoplastic dermatomyositis with histopathologic changes confined to the acrosyringia. Am J Dermatopathol 2017; 39: 3-7.

7. Durieux V, Coureau M, Meert AP, et al. Autoimmune paraneoplastic syndromes associated to lung cancer: A systematic review of the literature. Lung Cancer 2017; 106: 102-9.
8. Aggarwal R, Oddis CV. Paraneoplastic myalgias and myositis. Rheum Dis Clin North Am 2011; 37: 607-21.

9. Kanaji N, Watanabe N, Kita N, et al. Paraneoplastic syndromes associated with lung cancer. World J Clin Oncol 2014; 5: 197-223.

10. Lange U, Bachmann G, Müller-Ladner U. Tibial pain and unilateral knee arthritis: Precursors of paraneoplastic arthropathy. Z Rheumatol 2014; 70: 332-5.

11. Paraschiv B, Diaconu CC, Toma CL, Bogdan MA. Paraneoplastic syndromes: the way to an early diagnosis of lung cancer. Pneumologia 2015; 64: 14-9.

12. Miret M, Horváth-Puhó E, Déruaz-Luyet A, et al. Potential paraneoplastic syndromes and selected autoimmune conditions in patients with non-small cell lung cancer and small cell lung cancer: A population-based cohort study. PLoS One 2017; 12: 0181564.

13. Pileckyte M, Baliūnaite R, Tamulaitiene V. Paraneoplastic rheumatic syndromes. Medicina 2018; 39: 443-7.

14. Hakkou J, Rostom S, Bahiri R, Hajjaj-Hassouni N. Paraneoplastic rheumatic syndromes: report of eight cases and review of literature. Rheumatol Int 2012; 32: 1485-9.

15. Stummvoll GH, Aringer M, Machold KP, et al. Cancer polyarthritis resembling rheumatoid arthritis as a first sign of hidden neoplasms. Report of two cases and review of the literature. Scand J Rheumatol 2010; 30: 40-4.

16. Pourmorteza M, Baumrucker SJ, Al-Sheyyab A, Da Silva MA. Hypertrophic pulmonary osteoarthropathy: a rare but treatable condition in palliative medicine. J Pain Symptom Manage 2015; 50: 263-7.

17. Callemeyn J, Van Haecke P, Peetermans WE, Blockmans D. Clubbing and hypertrophic osteoarthropathy: insights in diagnosis, pathophysiology, and clinical significance. Acta Clin Belg 2016; 71: 123-30.

18. Kieffer JM, Postma TJ, van de Poll-Franse L, et al. Evaluation of the psychometric properties of the EORTC chemotherapy-induced peripheral neuropathy questionnaire (QLQ-CIPN20). Qual Life Res 2017; 20: 172-8.

19. Kolb NA, Smith AG, Singleton JR, et al. The association of chemotherapy-induced peripheral neuropathy symptoms and the risk of falling. JAMA Neurol 2016; 73: 860-6. 